\title{
Experimental Chatter Characterization in Metal Band Sawing
}

\author{
Tilen Thaler ${ }^{1}{ }^{*}$ - Primož Potočnik ${ }^{2}$ - Janez Kopač ${ }^{2}$ - Edvard Govekar ${ }^{2}$ \\ 1 PETRA stroji d.o.o., Slovenia \\ 2 University of Ljubljana, Faculty of Mechanical Engineering, Slovenia
}

One of the most detrimental instability phenomena in metal band sawing is chatter, i.e. high amplitude vibrations of the tool and/or workpiece. In this paper, the influence of the cutting speed and of the distance between the blade supports on chatter phenomena is investigated. For this purpose a series of experiments with triangular cutting speed variation at several pre-selected distances between the blade supports was conducted on structural steel (St37, DIN 17100) workpieces. A feature for chatter detection was extracted from the power spectra of the machine vibration signal, and a set of characteristics was introduced for experimental chatter characterization. The results showed the presence of a chatter hysteresis which depended on the cutting speed. Additionally, apart from the blade support distance, the cutting speed was shown to be a strongly influencing parameter, and as such also promising for chatter control in band sawing processes.

Keywords: chatter, metal band sawing, empirical characterization

\section{INTRODUCTION}

In cutting processes, chatter can be characterized as the self-excited, high amplitude vibrations of the cutting blade and/or workpiece [1]. It is caused by the instability which occurs in a nonlinear cutting process, and can result in harmful effects on process performance from the point of view of quality, economy, and ecology [1] and [2]. The chatter phenomenon has been intensively researched in finalizing machining operations such as turning [3] and milling (removal rate maximization and chatter suppression) [4] and [5]. Grinding is also highly susceptible to the occurrence of chatter, so several researchers have contributed to the understanding of the chatter phenomenon by non-linear coarse-grained entropy rate analysis [6], non-linear modelling of the grinding process and chatter prediction [7], as well as chatter detection [8] and stability lobe prediction [9].

As opposed to these machining operations, band sawing is most often used at the beginning of the machining chain, and as such does not influence the final product properties as significantly as the finalizing processes. However, with the development of expensive and sometimes difficult-to-cut metal alloys and other materials, such as mono-crystalline silicon, minimization of waste material and of the time for final machining operations, as well as maximisation of the corresponding surface quality, have become increasingly important.

In band sawing, the negative impact of chatter on the quality of products was first noticed in lumber cutting [10] and [11], and was reflected in the so-called wash-boarding [10] of the cut surface. To be able to understand, predict and avoid chatter vibrations in band sawing, several mathematical models were derived.
Their aim was to predict the natural frequencies of the moving continuum, representing the band saw blade. In order to predict the natural frequencies, which are related to the chatter phenomenon, models based on bending [10] and torsional deformations [11] were developed, as well as several models that were based on a moving plate with respect to tangential loading [12], parametric excitation [13], and non-conservative force excitation [14]. Experimental studies of the band saw blade vibrations showed that tensioning of the blade increases the fundamental torsional frequency, but does not affect the lateral bending frequency [15]. Current experimental and model-based solutions for chatter avoidance in band sawing suggest control of the cutting speed [10] and [16], avoidance of excitation at the natural frequencies of the cutting blade, and maximizing the tension of the cutting blade [15]. However, the relevance of complex, theoretical models based on band sawing process characterization is determined, to a high degree, by the approximations of the material characteristics, friction and temperature effects in the cutting zone, all of which are non-linear. For this reason in this research the experimental characterization was chosen for chatter characterization. In our previous research, several features based on spectral analysis were defined and successfully applied for chatter detection in band sawing [16]. The aim of this paper is to apply the most relevant chatter detection feature [16] for the characterization of chatter and related chatter hysteresis. Information on chatter and related chatter hysteresis characteristics is important for the implementation of chatter control and process optimization in the near-chatter regions.

In the following section, the experimental setup and experiments are described. Band sawing 
experiments with different distances between the blade supports $L_{b}$ and varying cutting speeds $v_{c}$ were performed in order to collect experimental data about chatter. In the section: "Analysis and characterization of chatter", the most informative feature for chatter detection is defined as the basis for further analysis. It is applied to the acquired experimental data, and the results are presented, indicating the strong influence of the cutting speed and the distance between the blade supports on onset of chatter and the characteristics of the observed chatter hysteresis. Finally, the results are summarized in the conclusions, where the most significant contribution of this work is presented and possible implementation issues in band sawing are discussed.

\section{EXPERIMENTAL SETUP}

The cutting experiments were conducted on a double column PE-TRA Toolmaster 300DC band saw with a $300 \mathrm{~mm}$ maximum cutting width. The maximum cutting width is defined by the distance between the vertical cutting blade supports $L_{b}$, as shown in Fig.1 right. This distance $L_{b}$ can be pre-set within the range from 230 to $420 \mathrm{~mm}$. A bimetal cutting blade of length $4150 \mathrm{~mm}$ was tensioned by $2.0 \mathrm{kN}$. The characteristic parameters of the cutting blade are given in Table 1.

Table 1. Cutting blade parameters

\begin{tabular}{ll}
\hline Parameter & Value \\
\hline Material & M42 \\
\hline Loop length & $4150[\mathrm{~mm}]$ \\
\hline Width/Thickness & $34 / 1.1[\mathrm{~mm}]$ \\
\hline Teeth pitch & 2 to $3[$ teeth per inch] \\
\hline Rake/Clearance angle & $10 / 32\left[^{\circ}\right]$ \\
\hline
\end{tabular}

In order to measure and control the cutting speed $v_{c}$, the band saw machine is equipped with a frequency inverter, a controller, and software for controlled variation of the cutting speed.

A three-component Kistler 9257B dynamometer, a three-component PCB 356A16 piezo accelerometer, a Brüel \& Kjaer 4190 microphone, a preamplifier 2669 , and an amplifier 2691-OS1 were used to detect cutting forces, machine vibrations and sound emitted by the cutting process. The frequency responses of the dynamometer and accelerometer were, in terms of their first natural frequency and according to the manufacturers, 2 and $10 \mathrm{kHz}$ respectively for the applied type of mounting. The microphone frequency response was $\pm 2 \mathrm{~dB}$ in the frequency range $3.15 \mathrm{~Hz}$ to $20 \mathrm{kHz}$. Placement of the sensors is shown in Fig. 1. The workpiece was mounted on the threecomponent dynamometer, in order to measure the cutting force components $\boldsymbol{F}=\left(F_{f}, F_{c}, F_{l}\right)$. The subscripts $c, f$, and $l$ denote the components in the cutting, feed, and lateral directions. In order to measure the machine vibrations $\boldsymbol{a}=\left(a_{f}, a_{c}, a_{l}\right)$ the three-component accelerometer was mounted on the left cutting blade support. The blade supports are in direct contact with the cutting blade and represent one of the most dynamically exposed parts of the machine structure. The emitted sound pressure $p$ was measured by the microphone, which was positioned $32 \mathrm{~cm}$ above the workpiece and directed toward the cutting zone.

All the sensory data obtained during the band sawing process were acquired by a 16bit resolution A/D data acquisition system, and were transferred to a computer for off-line analysis and chatter characterisation. The sampling frequencies for the cutting force components $\left(F_{f}, F_{c}, F_{l}\right)$, the machine
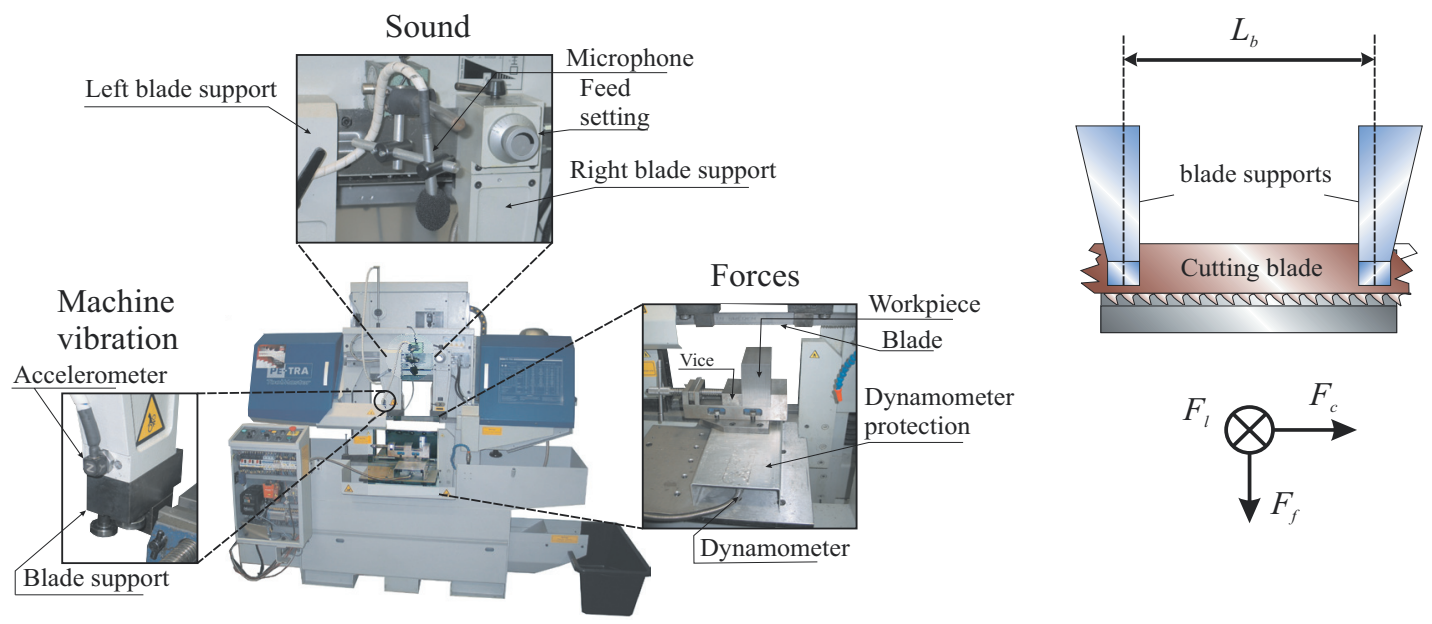

Fig. 1. Double-column horizontal band saw experimental set-up 
vibration $\left(a_{f}, a_{c}, a_{l}\right)$, and the sound pressure $p$ signals, were $20,25.6$ and $20 \mathrm{kHz}$, respectively.

\subsection{Experiments}

The experimental set-up on the multi-sensory double column horizontal band saw provided the basis for the chatter characterization. In the band sawing experiments, rectangular solid profile workpieces with a width of $100 \mathrm{~mm}$ and a height of $60 \mathrm{~mm}$, made of structural steel type St37 (according to DIN 17100), were used. The main control parameters of the experiments were the cutting speed $v_{c}$ and the distance between the blade supports $L_{b}$.

In order to investigate the influence of the cutting speed $v_{c}(t)$ on the band sawing process dynamics and the related chatter, the cutting speed $v_{c}$ was varied in a triangular manner as shown in Fig. 2a. The experiments started with the cutting speed $v_{c}$ set to $34 \mathrm{~m} / \mathrm{min}$. During the cutting the $v_{c}$ was linearly increased so that in 30 seconds it reached maximal value $133 \mathrm{~m} / \mathrm{min}$. After a second the cutting speed was decreased at a constant rate so that the minimal value of $34 \mathrm{~m} / \mathrm{min}$ was again reached in 30 seconds, and the cutting experiment, at the preset distance between the blade supports $L_{b}$, was stopped.

To investigate the influence of the distance between the blade supports $L_{b}$ the signals of the cutting forces $\boldsymbol{F}$, accelerations $\boldsymbol{a}$, and sound pressure $p$ were acquired during a set of 35 experiments at seven preset distances between the blade supports $L_{b}=[250, \ldots, 400] \mathrm{mm}$ with increments of $25 \mathrm{~mm}$. At each preset distance between the blade supports, 5 experiments were performed, which resulted in the total number of 35 experiments. The number of 5 repeated experiments was chosen as a compromise between achieving sufficient statistical significance of the acquired data, and minimizing the wear of the cutting edges of the blade during the complete set of experiments.

The process parameters depend on the selected workpiece material and are recommended by the cutting blade manufacturer. Based on this, the experiments were performed at feed rate of $v_{f}=45 \mathrm{~mm} / \mathrm{min}$. At this feed rate, the feed component of the cutting force $F_{f}$ was within the recommended limits, i.e. less than $70 \mathrm{~N}$ per cutting tooth of the band saw blade. The corresponding cutting rate was $100 \mathrm{~mm}^{2} / \mathrm{s}$.

Examples of the acquired signals of feed force $F_{f}$, the acceleration in the cutting direction $a_{c}$, and the sound pressure $p$ measured during the band sawing of the workpieces at a preset distance between the blade supports of $L_{b}=350 \mathrm{~mm}$, are shown in Fig. 2 $\mathrm{b}, \mathrm{c}$ and $\mathrm{d}$. In the presented signals the regions of low amplitude oscillations related to the regular chatterfree cutting are marked in blue, and the regions of large amplitude vibrations related to the cutting by chatter are marked in red.

More in detail, during the linear increase of the cutting speed $v_{c}$ an abrupt increase in the amplitudes of the acquired signal $a_{c}$ can be observed at time $t \approx 9.7 \mathrm{~s}$ and a cutting speed of $v_{c} \approx 67.1 \mathrm{~m} / \mathrm{min}$ respectively. The large amplitude oscillations terminate at $\mathrm{t} \approx 12.5 \mathrm{~s}$ and are re-excited at $t \approx 19.5 \mathrm{~s}$ and $v_{c} \approx 97.3 \mathrm{~m} / \mathrm{min}$. The excited high amplitude oscillations remain present until $t \approx 41.3 \mathrm{~s}$, when the cutting speed $v_{c}$ is decreased to $v_{c} \approx 100.2 \mathrm{~m} / \mathrm{min}$. Similarly as during the increase of the cutting speed $v_{c}$ large-amplitude oscillations of short duration are excited again at time $t \approx 48.6 \mathrm{~s}$ and $v_{c} \approx 75.1 \mathrm{~m} / \mathrm{min}$.

The observed high amplitude vibrations at $v_{c} \approx 67.1,97.3$ and $75.1 \mathrm{~m} / \mathrm{min}$ are caused by the instability of the regular chatter free cutting, where an abrupt transition to chatter takes place. Additionally, a small cutting velocity hysteresis can be observed with respect to the first chatter onset at $v_{c o} \approx 67.1 \mathrm{~m} / \mathrm{min}$ and the last chatter termination velocity $v_{c t} \approx 66.8 \mathrm{~m} / \mathrm{min}$. This observation, together with the observed abrupt changes in the oscillation amplitude, indicates that band sawing process instability and related chatter phenomena can be described by sub-critical Hopf bifurcation [1].

\section{ANALYSIS AND CHARACTERIZATION OF CHATTER}

In general it has been observed that all of the acquired signals appear to be informative with respect to chatter [16]. However, a time-dependent mean value of the feed force component can be observed due to the change in the cutting speed and the effects of the workpiece geometry [17] and [18]. The mean value of the feed force is proportional to the number of the teeth in the cut and the depth of the cut [18]. Thus, the geometry of the workpiece, as well as the cutting speed variation, causes the non-stationarity of the feed force component observed in Fig. 2. In the acceleration and sound measurements there are no evidences of the workpiece geometry influence. Accelerometers also have several on-site advantages compared to the dynamometers and microphones, so the acceleration signal in the cutting direction ac was used in the following analysis for the characterization of chatter in band sawing. 

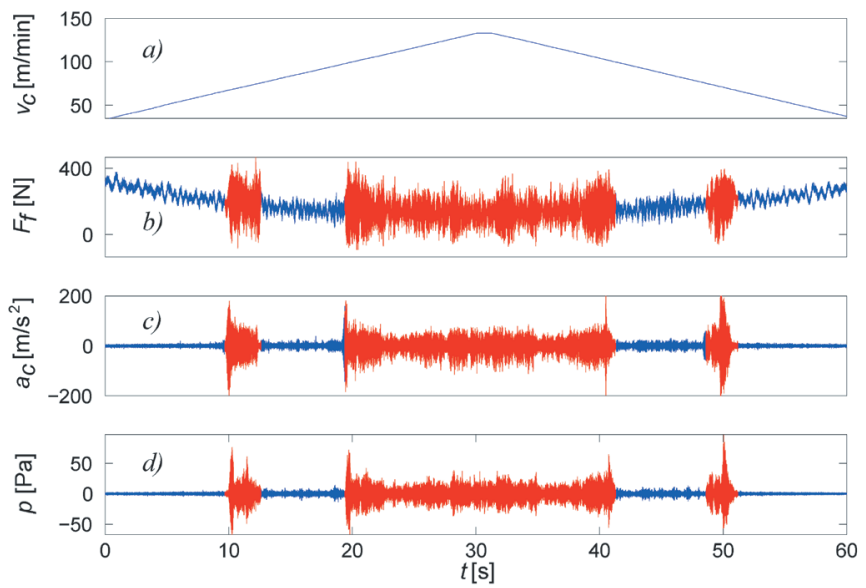

Fig. 2. a) the cutting speed $v_{c}(t)$ triangular profile; examples of the corresponding acquired signals at the blade support distance of $L_{b}=350$ $\mathrm{mm} ; b)$ feed force $\left.F_{f}, c\right)$ the acceleration in the cutting direction $a_{c}$ and, d) the sound pressure $p$; low amplitude regular cutting is marked in blue and large amplitude chatter cutting is marked in red
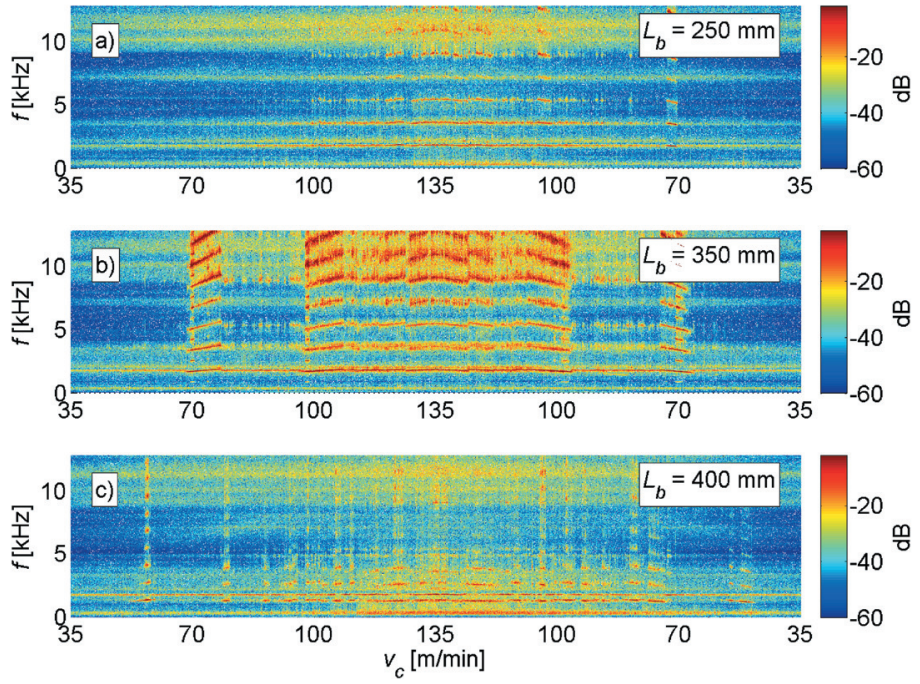

Fig. 3. Spectrogram of the acceleration signal $a_{c}$ versus the cutting speed $v_{c}$ at three different distances between the blade supports $L_{b}$

\subsection{Data Analysis}

Chatter in cutting is reflected in high amplitude oscillation of the feed force $F_{f}$, the acceleration in the cutting direction $a_{c}$, and the sound pressure $p$ were presented with indicated chatter regions (Figs. 2b, c and d). Since chatter is a dynamical phenomenon, apart from the observed chatter-related high amplitude vibrations, chatter can be more precisely characterized by changes and amplification of the power spectra components at the specific harmonic frequencies [16], [19] and [20] in the power spectra of the acquired signals. Fig. 3 shows examples of spectrograms of the acceleration signal in the cutting direction $a_{c}$ acquired during the band sawing process, with variation of the cutting speed $v_{c}$ performed at three different distances between the blade supports, i.e. $L_{b}=250,350$ and 400 $\mathrm{mm}$. The pronounced horizontal bands of amplified frequencies in the spectrograms were found to be characteristic for chatter cutting, whereas a relatively uniform, low power frequency pattern is characteristic for the regular chatter-free cutting. Based on the frequency pattern observed in the spectrograms we can see that chatter is the most present at the distance $L_{b}=350 \mathrm{~mm}$ what indicates a nonlinear influence of distance $L_{b}$ on chatter in band sawing.

Typical $a_{c}$ signal power spectra of the chatter and chatter free cutting regimes are shown in Fig. 4. As already evident from the spectrograms in the chatter 

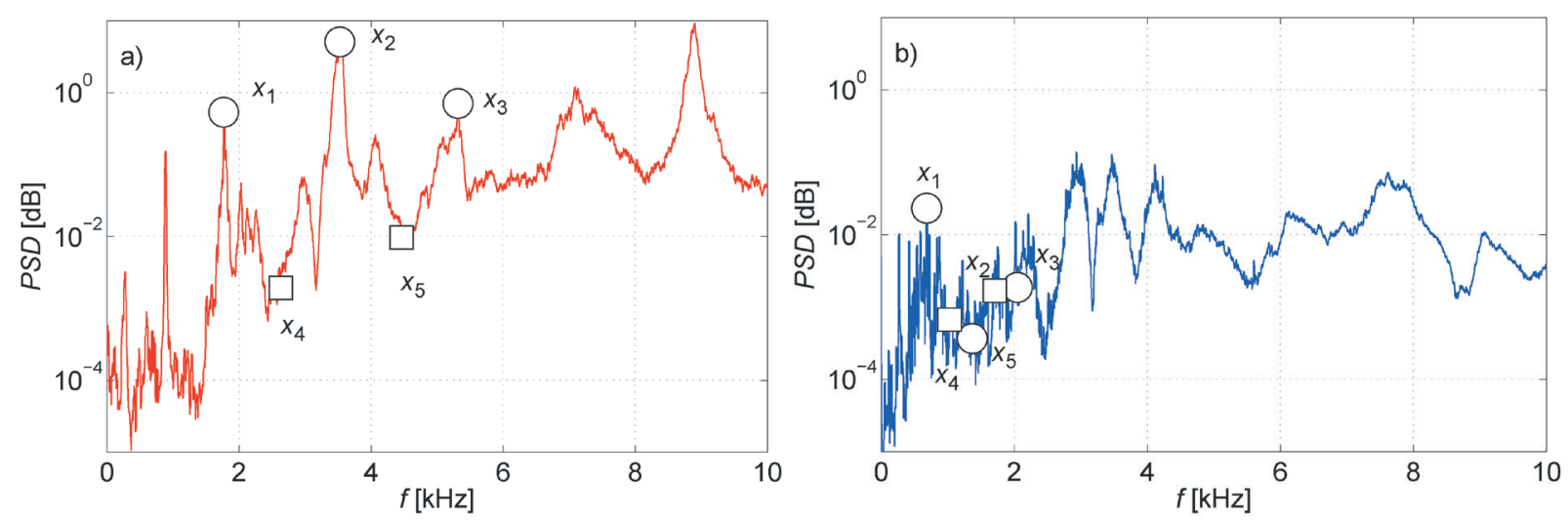

Fig. 4. Characteristic power spectra and defined basic power spectra features $x_{1} \ldots x_{5}$ of the acceleration signal $a_{c}$ in; a) chatter and, b) chatter-free cutting

power spectrum (Fig. 4a) pronounced peaks at certain frequencies are characteristic.

For more detailed analysis and chatter characterisation a quantitative power spectra chatter feature $y$ was defined as a linear combination of the basic normalized power spectra features $y[16]$ :

$$
y=x_{1}+x_{2}+x_{3}-x_{4}-x_{5} .
$$

The basic power spectra features $x_{1}, \ldots, x_{5}$ are denoted in Fig. 4 and are defined as:

$x_{1}$ : amplitude of the $1^{\text {st }}$ maximal peak in the frequency range $[0,2] \mathrm{kHz}$,

$x_{2}$ : amplitude of the $2^{\text {nd }}$ harmonic peak,

$x_{3}$ : amplitude of the $3^{\text {rd }}$ harmonic peak,

$x_{4}$ : amplitude in the middle between the $1^{\text {st }}$ and the $2^{\text {nd }}$ harmonic peak,

$x_{5}$ : amplitude in the middle between the $2^{\text {nd }}$ and the $3^{\text {rd }}$ harmonic peak.

The defined feature $y$ characterizes the properties of the power spectra as they are related to chatter and chatter-free cutting. The feature's value is high for spectra with a high peak-to-valley difference, which is generally the case in the chatter regime with more deterministic signals. On the other hand the value of the feature $y$ is low for regular cutting regimes, whose signals resemble a stochastic process.

\subsection{Results}

Fig. 5 shows the values of the feature $y$ of the acceleration signal $a_{c}$ in dependence of the cutting speed $v_{c}$ during the increasing of the cutting speed (blue curves), and during the decreasing of the cutting speed (red curves), for five repetitions of the cutting experiment at a distance between the blade supports of $L_{b}=350 \mathrm{~mm}$.

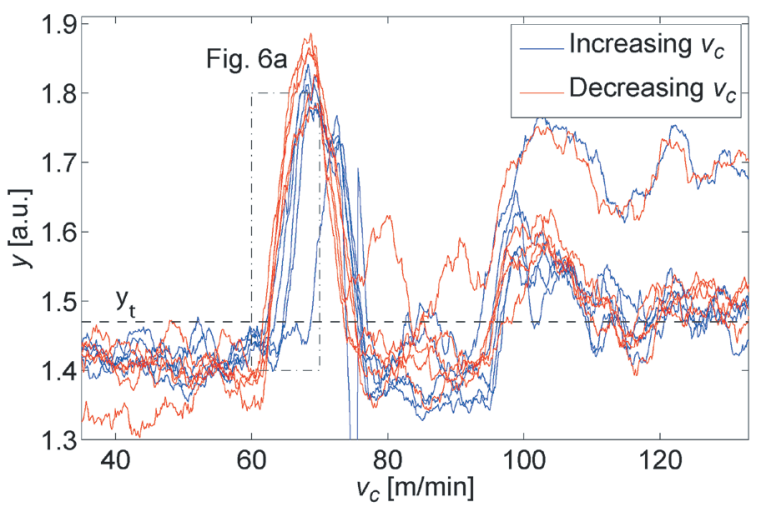

Fig. 5. Feature y plotted against the increasing (blue) and decreasing (red) cutting speed $v_{c}$, and corresponding chatter threshold $y_{t}=1.47$

The feature $y$ maintains relatively low values in chatter free cutting regime until the cutting speed reaches a value of approximately $v_{c} \approx 61 \mathrm{~m} / \mathrm{min}$. At this breakpoint cutting speed $v_{c}, y$ starts to increase rapidly. This rapid increase in $y$ is caused by the transition from chatter free to chatter cutting. The threshold value $y_{t}$ of the feature $y$ which indicates the onset of chatter cutting, was defined as an average value of the feature values $y$ at the breakpoint cutting speed $v_{c}$ In Fig. 5 the calculated chatter threshold value $y_{t}=1.47$ is denoted by a dashed horizontal line. Thus values of the feature $y$ which lie below and above the threshold line $y_{t}$ indicate chatter-free and chatter cutting respectively. The high values of $y$ in the interval around $v_{c} \approx 70 \mathrm{~m} / \mathrm{min}$ and $v_{c} \approx 104 \mathrm{~m} / \mathrm{min}$ are indicators of strong chatter.

A closer inspection of the feature $y$ in the region around the breakpoint is provided in Fig. 6a, where the values of the feature $y$ during increasing (solid) and decreasing (dashed) cutting speed $v_{c}$ can be seen. It is 

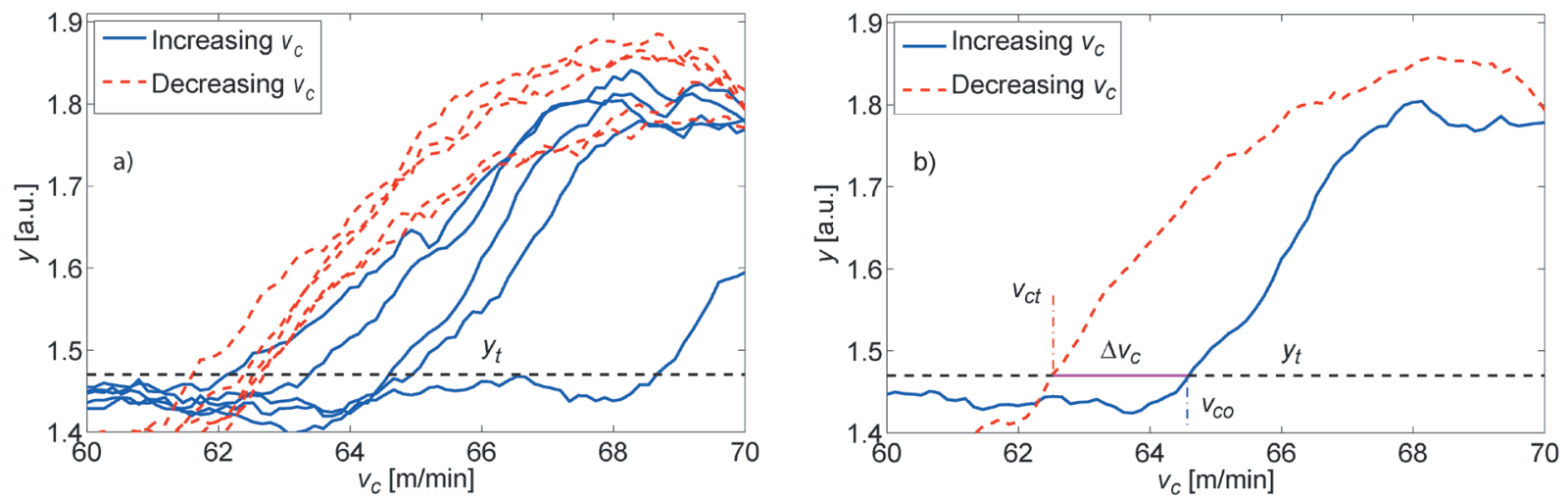

Fig. 6. a) Detailed view of the feature $y$ vs. increasing (solid) and decreasing (dashed) cutting speed $v_{c}$ and corresponding threshold value $y_{t}$. b) Mean values of the feature $y$ and defined chatter hysteresis characteristics with respect to the threshold value $y_{t}$

evident from the Fig. 5 that in the case of increasing cutting speed, the feature $y$ on average crosses the threshold value $y_{t}$ at higher cutting speeds $v_{c}$, than in the case of decreasing cutting speed. This indicates the presence of a hysteresis in the band sawing process with respect to the cutting speed $v_{c}$, which is more clearly observed in the plot of the feature $y$ mean value during the increasing (solid) and decreasing (dashed) cutting speed $v_{c}$, shown in Fig. 6b. The presence of the hysteresis further indicates that, in the case of the band sawing process, the instability, which causes the onset of chatter, is the same as that observed in turning processes, and can be attributed to sub-critical Hopf bifurcation [1].

For further analysis and characterization of the observed chatter hysteresis, several characteristics as shown in Fig. $6 \mathrm{~b}$ were defined. The characteristic of chatter onset $v_{c o}$ was defined by the cutting speed $v_{c}$ at which the feature $y$ (solid) at increase of $v_{c}$ crosses the threshold value $y_{t}$. Similarly the characteristic of chatter termination cutting speed $v_{c t}$ was defined by the cutting speed $v_{c}$ at which the feature $y$ (dashed) at decrease of $v_{c}$ crosses the threshold value $y_{t}$.

Table 2. Chatter characteristics

\begin{tabular}{lc}
\hline Characteristic & Symbol \\
\hline Chatter onset cutting speed $[\mathrm{m} / \mathrm{min}]$ & $v_{c 0}$ \\
\hline Chatter termination cutting speed $[\mathrm{m} / \mathrm{min}]$ & $v_{c t}$ \\
\hline Chatter hysteresis width $[\mathrm{m} / \mathrm{min}]$ & $\Delta v_{c}$ \\
\hline Relative chatter duration $[\%]$ & $t$ \\
\hline
\end{tabular}

By the difference of the chatter onset $v_{c o}$ and chatter die-out $v_{c t}$ cutting speeds, the width of the chatter hysteresis $\Delta v_{c}$ was defined. Further, as an additional chatter characteristic, the relative chatter duration $\tau$, was defined as the ratio between chatter duration and the duration of the entire experiment which lasted 60 seconds. The proposed set of chatter characteristics is presented in Table 2 .

In the following the dependence of the defined characteristics versus the distances $L_{b}$ of the cutting blade supports are shown by mean of the box plots.

The box plots were obtained based on 5 experiments of cutting speed variation at each considered distance $L_{b}$. The central horizontal line in the box indicates the median, whereas the upper and lower box boundaries are located at the $1^{\text {st }}$ and $3^{\text {rd }}$ quartiles respectively, with whiskers placed at 3-times the standard deviation of the sample, and single points $(+)$ outside the whiskers representing outliers.

In Fig. 7, the box plot of chatter onset $v_{c o}$, (solid) and chatter termination cutting speed $v_{c t}$, (dashed) against the distance between the blade supports $L_{b}$ are shown.

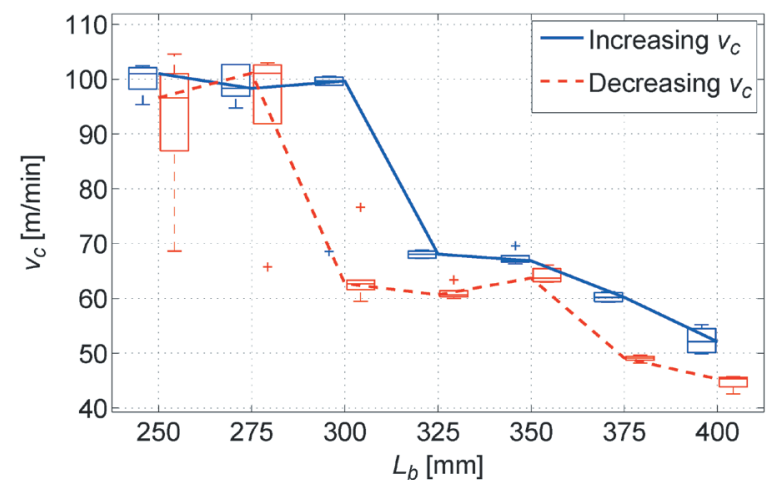

Fig. 7. Box plot of the chatter onset $v_{c o}$ (solid) and chatter termination $v_{c t}$ cutting speeds (dashed) vs. the distance between the blade supports $L_{b}$

From the box plot (Fig. 7) it can be seen that both the chatter onset and chatter termination median cutting speeds are around $100 \mathrm{~m} / \mathrm{min}$ for the first two shorter distances $L_{b}$. At a distance of $L_{b}=300 \mathrm{~mm}$, 
the median chatter termination cutting speed drops to $v_{c t}=62.4 \mathrm{~m} / \mathrm{min}$, whereas the median chatter onset cutting speed remains at $v_{c o}=100 \mathrm{~m} / \mathrm{min}$. With a further increase in the distance to $L_{b}=325 \mathrm{~mm}$, the median cutting speed of chatter onset drops to approximately $v_{c o}=68.1 \mathrm{~m} / \mathrm{min}$, and further decreases to a value of $v_{c o}=53.4 \mathrm{~m} / \mathrm{min}$ at a distance of $L_{b}=$ $400 \mathrm{~mm}$. The median chatter onset cutting speeds $v_{c o}$ are always somewhat higher than the corresponding chatter termination cutting speeds $v_{c t}$, represented by the solid and dashed lines respectively. The connecting lines of the median chatter onset and termination speeds reveal the chatter hysteresis at all distances between the blade supports where $L_{b}>275 \mathrm{~mm}$.

The influence of the distance between the blade supports $L_{b}$ on the width of the chatter hysteresis $\Delta v_{c}$ is shown in Fig. 8.

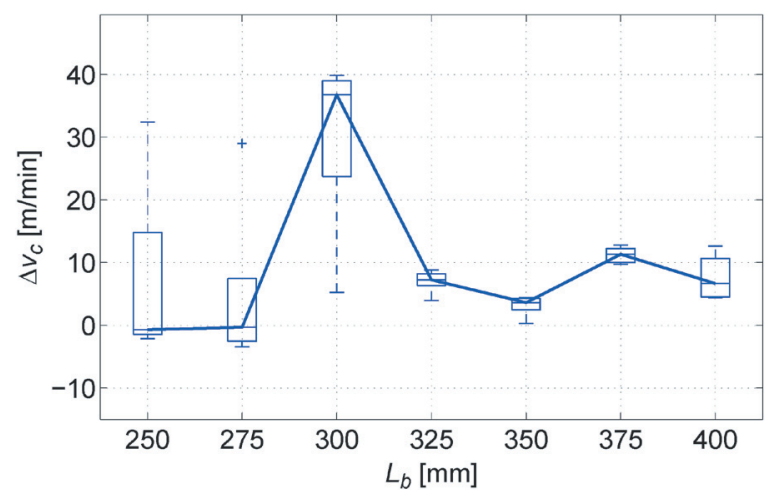

Fig. 8. Width of the cutting speed hysteresis $\Delta v_{c}$ vs. the blade support distance $L_{b}$

In the case of distances $L_{b} \leq 275 \mathrm{~mm}$, the median values of $\Delta v_{c}$ are close to zero and hysteresis cannot be observed. The largest median hysteresis width $\Delta v_{c}=34.8 \mathrm{~m} / \mathrm{min}$ occurs at a blade support distance of $L_{b}=300 \mathrm{~mm}$, denoting the largest bi-stable region of the band sawing process. With further increases in the blade support distance $L_{b}$, the length of the cutting speed hysteresis $\Delta v_{c}$ decreases, and scatters around $\Delta v_{c}=8$ to $10 \mathrm{~m} / \mathrm{min}$. The observed cutting speed differences $\Delta v_{c}$ between the chatter onset $v_{c o}$ and chatter termination $v_{c t}$ cutting speeds, as they depend on the blade support distance $L_{b}$, additionally confirm the presence of the hysteresis, which is characteristic for the onset of a non-linear chatter phenomenon in cutting [1] and [21].

The box plot of the relative chatter duration $\tau$ against the distance between the blade supports $L_{b}$ is presented in Fig. 9. Considering the median values of $\tau$, it can be seen from Fig. 9 that the relative chatter duration $\tau$ is short in the case of short distances $L_{b}$, and is not affected by the distance $L_{b}$. In case of distances $L_{b}>275 \mathrm{~mm}$, the relative duration of the chatter $\tau$ increases as the blade support distance $L_{b}$ increases.

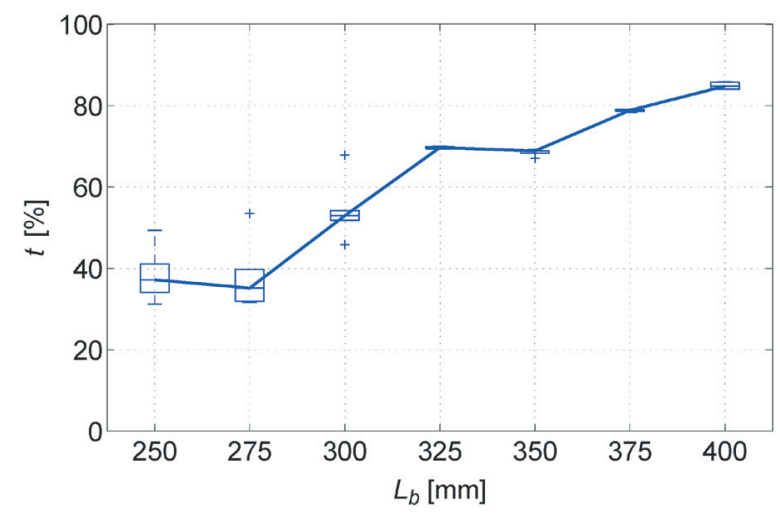

Fig. 9. Relative duration of chatter $T$ vs. the blade support distance $L_{b}$

The longest relative duration of the chatter $\tau=85 \%$ took place in the case of the largest blade support distance $L_{b}=400 \mathrm{~mm}$.

\section{CONCLUSIONS}

In the paper the characterization of chatter phenomena in metal band sawing process is considered. This characterization is based on an analysis of the acquired acceleration signals in the cutting direction $a_{c}$ during the band sawing process. In particular, the influence of the cutting speed $v_{c}$ and of the distance between the blade supports $L_{b}$ on chatter occurrence were investigated. For chatter detection a feature is extracted from the power spectra of the machine vibration signal, and a set of characteristics is introduced for chatter characterization. The set of characteristics beside the relative chatter duration $t$ includes: chatter onset cutting speed $v_{c o}$, chatter termination cutting speed $v_{c t}$, and the width of the cutting speed hysteresis $\Delta v_{c}$.

Based on the analysis of the influence of the cutting speed $v_{c}$, the following conclusion can be drawn:

- In general, chatter onset takes place at higher cutting speeds $v_{c o}$, and terminates at lower cutting speeds $v_{c t}$. The observed cutting speed differences $\Delta v_{c}$ between the chatter onset and chatter termination cutting speeds indicate the presence of hysteresis, which is characteristic for subcritical Hopf bifurcation based instability of the cutting process and the related onset of the chatter phenomenon in cutting. 
- The results of the analysis of the influence of the distance between the cutting blade supports $L_{b}$ show that:

- With an increase in the distance between the cutting blade supports $L_{b}$ there is a decrease in both the chatter onset cutting speed $v_{c o}$ and in the chatter termination cutting speed $v_{c t}$.

- The relative chatter duration $\tau$ increases with the increase of the distance between the cutting blade supports $L_{b}$.

The observed influence of the distance between the blade supports on the chatter onset speed vco and the chatter termination speed vct as well as on the relative chatter duration $\tau$, suggest that the blade supports should be as close to the workpiece as possible.

Furthermore, apart from the blade support distance $L_{b}$, which is defined by the geometry of the workpiece, the cutting speed $v_{c}$ is shown to be a promising parameter for chatter control in the band sawing processes, with the potential for optimal cutting in near-chatter regions. Although not considered in the paper, it can be expected that the type of material under test, as well as the tool material and wear, will have a significant effect on the chatter onset and hysteresis characteristics.

\section{ACKNOWLEDGEMENT}

The operation was partly financed by the European Union and European Social Fund. Operation, which was implemented within the framework of the Operational Program for Human Resources Development for the period 2007 to 2013, Priority axis 1: Promoting entrepreneurship and adaptability, Main type of activity 1.1.: Experts and researchers for competitive enterprises.

\section{REFERENCES}

[1] Gradišek, J., Govekar, E., Grabec, I. (2001). Chatter onset in non-regenerative cutting: A numerical study. Journal of Sound and Vibration, vol. 242, no. 5, p. 829838, DOI:10.1006/jsvi.2000.3388.

[2] Quintana, G., Ciurana, J. (2011). Chatter in machining processes: A review. International Journal of Machine Tools and Manufacture, vol. 51, no. 5, p. 363-376, DOI:10.1016/j.ijmachtools.2011.01.001.

[3] Pušavec, F., Govekar, E., Kopač, J., Jawahir, I.S. (2011). The influence of cryogenic cooling on process stability in turning operations. CIRP Annals - Manufacturing Technology, vol. 60, no. 1, p. 101-104, DOI:10.1016/ j.cirp.2011.03.096.
[4] Budak, E., Tekeli, A. (2005). Maximizing chatter free material removal rate in milling through optimal selection of axial and radial depth of cut pairs. CIRP Annals - Manufacturing Technology, vol. 54, no. 1, p. 353-356, DOI:10.1016/S0007-8506(07)60121-8.

[5] Shamoto, E., Mori, T., Sencer, B., Suzuki, N., Hino, R. (2013). Suppression of regenerative chatter vibration in multiple milling utilizing speed difference method - Analysis of double-sided milling and its generalization to multiple milling operations. Precision Engineering, vol. 37, no. 3, p. 580-589, DOI:10.1016/j. precisioneng.2013.01.003.

[6] Govekar, E., Baus, A., Gradišek, J., Klocke, F., Grabec, I. (2002). A new method for chatter detection in grinding. CIRP Annals - Manufacturing Technology, vol. 51, no. 1, p. 267-270, DOI:10.1016/S00078506(07)61514-5.

[7] Robles-Ocampo, J.B., Jáuregui-Correa, J.C., Krajnik, P., Sevilla-Camacho, P.Y., Herrera-Ruiz, G. (2012). Nonlinear model for the instability detection in centerless grinding process. Strojniški vestnik - Journal of Mechanical Engineering, vol. 58, no. 12, p. 693-700, DOI:10.5545/sv-jme.2012.649.

[8] Ahrens, M., Fischer, R., Dagen, M., Denkena, M., Ortmaier, T. (2013). Abrasion monitoring and automatic chatter detection in cylindrical plunge grinding. Procedia CIRP, vol. 8, p. 374-378, DOI:10.1016/j. procir.2013.06.119.

[9] Ahmadi, K., Ismail, F. (2012). Stability lobes in milling including process damping and utilizing Multi-Frequency and Semi-Discretization Methods. International Journal of Machine Tools and Manufacture, vol. 54-55, p. 46-54, DOI:10.1016/ j.ijmachtools.2011.11.007.

[10] Mote, C.D. Jr. (1965). A study of band saw vibrations. Journal of the Franklin Institute, vol. 279, no. 6, p. 430-444, DOI:10.1016/0016-0032(65)90273-5.

[11] Alspaugh, D.W. (1967). Torsional vibration of a moving band. Journal of the Franklin Institute, vol. 283, no. 4, p. 328-338, DOI:10.1016/0016-0032(67)90047-6.

[12] Le-Ngoc, L., McCallion, H. (1995). Wide band saw blade under cutting conditions, part I: Stability of a plate moving in its plane while subjected to tangential edge loading. Journal of Sound and Vibration, vol. 186, no. 1, p. 125-142, DOI:10.1006/jsvi.1995.0438.

[13] Le-Ngoc, L., McCallion, H. (1995). Wide band saw blade under cutting conditions, part II: Stability of a plate moving in its plane while subjected to parametric cutting forces. Journal of Sound and Vibration, vol. 186, no. 1, p. 143-162, DOI:10.1006/jsvi.1995.0439.

[14] Le-Ngoc, L., McCallion, H. (1995) Wide band saw blade under cutting conditions, part III: Stability of a plate moving in its plane while subjected to nonconservative cutting forces. Journal of Sound and Vibration, vol. 186, no. 1, p. 163-179, DOI:10.1006/ jsvi.1995.0440.

[15] Kirbach, E., Bonac, T. (1978). The effect of tensioning and wheel tilting on the torsional and lateral 
fundamental frequencies of band saw blades. Wood and Fiber Science, vol. 9, no. 4, p. 245-251, from: http:// swst.metapress.com/content/A36J7007K0120U41, accessed on 2013-07-07.

[16] Thaler, T., Potočnik, P., Bric, I., Govekar, E. (2014). Chatter detection in band sawing based on discriminant analysis of sound features. Applied Acoustics, vol. 77, p. 114-121, DOI:10.1016/j.apacoust.2012.12.004.

[17] Thaler, T., Potočnik, P., Mužič, P., Bric, I., Bric, R., Govekar, E. (2012). Characterization of band sawing based on cutting forces. Journal of Machine Engineering, vol. 12, no. 1, p. 41-54.

[18] Ahmad, M.M., Hogan, B., Goode, E. (1988). Effect of machining parameters and workpiece shape on band sawing process. International Journal of Machine Tools and Manufacture, vol. 29, no. 2, p. 173-183, DOI:10.1016/0890-6955(89)90029-1.

[19] Rahman, M. (1986). Detection of the onset of chatter vibration. Journal of Sound and Vibration, vol. 109, no. 2, p. 193-205, DOI:10.1016/S0022-460X(86)80002-5.

[20] Delio, T., Tlusty, J., Smith, S. (1992). Use of audio signals for chatter detection and control. Journal of Engineering for Industry, vol. 114, no. 2, p. 146-157, DOI:10.1115/1.2899767.

[21] Strogatz, S.H. (1994). Nonlinear Dynamics and Chaos: with Applications to Physics, Biology, Chemistry, and Engineering (Studies in Nonlinearity). West View Press, Cambridge. 